\title{
Reconstruction of nasal ala and tip following skin cancer resection
}

Young Ji Park, Gyu Hyeon Kwon, Jun Oh Kim, Woo Sang Ryu, Kyung Suk Lee

Department of Plastic and Reconstructive Surgery, Gyeongsang National University Hospital, Jinju, Korea
Background: Defects of the nasal ala and tip have a complex three-dimensional structure that makes them challenging to reconstruct. Many reconstructive options have been described for nasal ala and tip defects, ranging from primary closure to local flaps and skin grafts. However, it is difficult to determine which method will yield the best cosmetic results in each individual case. Thus, the purpose of this study was to determine which surgical procedures for reconstructing defects of the nasal ala and tip have better cosmetic results.

Methods: From 2008 to 2018, 111 patients underwent surgery to reconstruct skin defects after resection of skin cancer in the nasal ala or tip. Their charts were reviewed to obtain data on age, sex, surgical location, size of the defect, surgical method, and cosmetic results using a visual analog scale (VAS).

Results: For nasal ala reconstruction, the most commonly used surgical technique was the nasolabial flap ( $\mathrm{n}=42)$. This method also had the highest VAS score (7/10). The most commonly selected surgical method for nasal tip reconstruction was the bilobed flap $(n=13)$, and bilobed flaps and primary closure had the highest VAS score (7/10).

Conclusion: Nasolabial flaps showed excellent cosmetic results for the reconstruction of nasal ala defects, while primary closure and bilobed flaps yielded excellent cosmetic results for the reconstruction of nasal tip defects.

Keywords: Nose / Skin neoplasm / Surgical flaps

\section{INTRODUCTION}

Defects may occur in the soft tissue around the nose due to various causes. However, the complex three-dimensional structure of defects of the nasal ala and tip make them difficult for surgeons to reconstruct. Various surgical techniques have been developed for the reconstruction of skin defects after resection of skin cancer on the nasal ala and tip [1]. Maintaining the lateral nasal wall and tip, with natural wrinkles of the nasal ala, is

\footnotetext{
Correspondence: Kyung Suk Lee

Department of Plastic and Reconstructive Surgery, Gyeongsang National

University Hospital, 79 Gangnam-ro, Jinju 52727, Korea

E-mail: opensound@hanmail.net

Received September 17, 2019/ Revised November 28, 2019/

Accepted November 29, 2019
}

integral for preserving the symmetry and the function of the nose. For these reasons, the choice of surgical procedure for nasal reconstruction depends on the location and size of the defect and the conditions of the patient's skin wrinkles.

The anatomical structure of the nose consists of the external skin, the internal nasal lining, the cartilaginous portion, and the fibrous fatty middle portion. Depending on the structures affected by a deformity, the most appropriate surgical procedure may range from a simple skin graft to a composite graft. The nasal ala and tip are more difficult to reconstruct than the internal nasal lining. Additionally, a cosmetic deformity may remain after nasal reconstruction. Small defects can be healed through secondary intention, but if the defect is large or if it involves most of the alar subunits, a local flap may result in poor cos- 
metic results due to pulling of the surrounding skin. Superficial skin defects can be reconstructed with full-thickness skin grafts, although problems such as scarring, an inappropriate contour, color mismatch, and the need for secondary reconstruction may occur [2].

For nasal reconstruction, local flaps (such as transposition flaps with a perforator, bilobed flaps, nasolabial turnover flaps, rotational flaps, and so forth) are widely used. Asymmetry may be caused by pulling on one side of the skin and blunting of the nasolabial angle, potentially requiring reoperation. Although many methods can be used for nasal reconstruction, it is difficult to determine the method that will have the best cosmetic results in specific cases. Thus, the purpose of this study was to determine which surgical procedures for reconstructing defects of the nasal ala and tip yield better cosmetic results.

\section{METHODS}

This study included patients who underwent surgery to reconstruct skin defects after resection of skin cancer on the nose at the Department of Plastic and Reconstructive Surgery of Gyeongsang National University Hospital from 2008 to 2018. All patients with single-area skin cancer that occurred in the nasal ala or tip were selected. Among the patients diagnosed with squamous cell carcinoma, melanoma, or basal cell carcinoma at the nasal ala and tip, the following more specific inclusion criteria were applied: skin cancer at a single location that was completely resected in the first operation, a resection margin confirmed to be free of cancer cells by frozen biopsy, and reconstruction with a single method. The charts, including postoperative photos, of the 111 patients who satisfied these criteria were reviewed to obtain data on age, sex, surgical location, size of the defect left after resection of the lesion, and method of surgery. By comparing photographs of the surgical site taken immediately after surgery with preoperative photos, the results of surgery were evaluated using a visual analog scale (VAS) by three plastic surgeons not involved in the operations.

Two patients underwent surgery under general anesthesia, while the remaining 109 patients received only local anesthesia. For local anesthesia, 1\% lidocaine with 1:100,000 epinephrine was used in the nose and cheeks. The criteria for determining the surgical resection margin generally followed the guidelines of the National Comprehensive Cancer Network [3,4]. However, if the skin cancer lesion was small or if the case was deemed to be low-risk, Mohs micrographic surgery was not performed. For basal cell carcinomas measuring less than $2 \mathrm{~cm}$, complete removal of the lesion was achieved with 4-mm peripheral surgical margins. For basal cell carcinomas larger than $2 \mathrm{~cm}$, the surgical margin was slightly larger than $4 \mathrm{~mm}$. Well-demarcated lesions, such as nodular basal cell carcinomas, were excised with a 3-mm margin. For almost all squamous cell carcinoma lesions, the surgical safety margin was $4 \mathrm{~mm}$; however, a surgical safety margin of $6 \mathrm{~mm}$ was used for high-risk lesions. In all cases, the resection margin was intraoperatively confirmed to be free of cancer cells via frozen biopsy.

After measuring the remaining defect size after resection of the skin cancer lesion, the surgical method for reconstruction was determined based on the location, depth, and size of the defect. The surgical methods used for reconstructions of the nasal ala and tip included bilobed flaps, local flaps (randomtype rotational or transposition flaps), nasolabial flaps, primary closure, skin grafts, forehead flaps, and free flaps. Of these methods, local flaps, nasolabial flaps, free flaps, and forehead flaps were classified as "other" flaps due to the low number of patients who underwent nasal tip operations using these methods. All harvested skin was full-thickness. A supraclavicular donor site was used in all cases where skin grafts were used. Defatting was performed. In all patients, a tie-over dressing was performed and replaced on the fifth day. In all patients who underwent flap surgery, the dressing was made using foam material. The subcutaneous layer was sutured with Vicryl 5-0 or polydioxanone 5-0, and the skin layer was sutured with blue nylon 6-0. By comparing photos taken immediately after surgery with photos taken before surgery, the quality of the reconstruction and cosmetic outcomes were evaluated by three plastic surgeons not involved in the operations using a VAS. The original VAS consisted of a $10-\mathrm{cm}$ line with the worst scar at the right end of the line and the best scar at the left end of the line. This VAS has been demonstrated to be reliable and valid for assessing cosmetic outcomes [5]. Therefore, in this study, VAS scores ranged from 0 to 10 , corresponding to their location on the $10-\mathrm{cm}$ line. Investigators marked the line at the point that they thought best corresponded to the surgical results.

To determine the significance of differences in patient data according to the surgical method, the Fisher exact test and the Kruskal-Wallis test were used. Additionally, differences in the distribution of defect size and VAS between surgical methods were tested using the Bonferroni correction. This study was approved by the Ethics Review Board of Gyeongsang National University Health Center (IRB No. 2019-08-0002).

\section{RESULTS}

In total, 111 patients were included in this study. There were 41 men and 70 women, with an average age of 71.8 years. Among them, 95 had basal cell carcinoma (ala, 60; tip, 35), 15 had squa- 
mous cell carcinoma (ala, 11; tip, 4), and one had melanoma (ala, 1). In the melanoma case, a black mass in the alar area suspected to be basal cell carcinoma was excised; however, it was diagnosed as benign melanoma based on the final biopsy results. All the skin cancer lesions were completely resected with a safety margin. Frozen biopsy confirmed that there were no residual cancer cells on the lateral and deep margins of the cut specimens. No complications such as seroma, wound dehiscence, or infection occurred after surgery. Seventy-two patients

Table 1. Demographic information and clinical findings of the patients enrolled in the study

\begin{tabular}{|c|c|c|c|c|}
\hline \multirow{2}{*}{ Variable } & \multirow{2}{*}{ Total $(n=111)$} & \multicolumn{2}{|c|}{ Location } & \multirow{2}{*}{$p$-value } \\
\hline & & Ala $(n=72)$ & $\operatorname{Tip}(n=39)$ & \\
\hline \multicolumn{4}{|l|}{ Operation type } & \multirow[t]{8}{*}{$<0.001^{\text {a) }}$} \\
\hline Bilobed & $16(14.41)$ & $3(4.17)$ & $13(33.33)$ & \\
\hline Local flap & $18(16.22)$ & $14(19.44)$ & $4(10.26)$ & \\
\hline Nasolabial flap & $44(39.64)$ & $42(58.33)$ & $2(5.13)$ & \\
\hline Primary closure & 18 (16.22) & $9(12.50)$ & $9(23.08)$ & \\
\hline Skin graft & $12(10.81)$ & $4(5.56)$ & $8(20.51)$ & \\
\hline Forehead flap & $2(1.80)$ & 0 & $2(5.13)$ & \\
\hline Free flap & $1(0.90)$ & 0 & $1(2.56)$ & \\
\hline \multicolumn{4}{|l|}{ Defect size (cm) } & \multirow[t]{4}{*}{$0.112^{\text {b) }}$} \\
\hline Mean \pm SD & $1.98 \pm 0.95$ & $2.07 \pm 0.91$ & $1.81 \pm 1.01$ & \\
\hline Median (IQR) & \multicolumn{3}{|c|}{$2.00(1.30,2.50) 2.20(1.50,2.50) 1.80(0.80,2.30)$} & \\
\hline (Min, Max) & $(0.50,6.00)$ & $(0.70,6.00)$ & $(0.50,5.50)$ & \\
\hline \multicolumn{4}{|l|}{ VAS } & \multirow[t]{4}{*}{$0.745^{\text {b) }}$} \\
\hline Mean \pm SD & $6.27 \pm 1.22$ & $6.25 \pm 1.22$ & $6.31 \pm 1.24$ & \\
\hline Median (IQR) & \multicolumn{3}{|c|}{$7.00(5.00,7.00) 6.50(5.00,7.00) 7.00(5.00,7.00)$} & \\
\hline (Min, Max) & $(3.00,9.00)$ & $(3.00,9.00)$ & $(3.00,8.00)$ & \\
\hline
\end{tabular}

Values are presented as number (\%).

SD, standard deviation; IQR, interquartile range; Min, minimum; Max, maximum; VAS, visual analog scale.

a)Fisher exact test; 'bilcoxon rank-sum test. underwent surgery to correct a nasal alar defect, with an average defect diameter of $2.07 \mathrm{~cm}$ and an average VAS score of 6.25. The other 39 patients underwent surgery to treat a nasal tip defect, with an average defect diameter of $1.81 \mathrm{~cm}$ and an average VAS score of 6.31 (Table 1).

The nasolabial flap was the most commonly used surgical technique for nasal ala reconstruction, followed by the local flap (random type). The average VAS score of bilobed flaps was 8, and the average VAS score of nasolabial flaps was 7. The average VAS score of skin grafts was 4 (Table 2). The choice of a local flap, primary closure, or a nasolabial flap as a surgical method showed a statistically significant difference according to the size of the defect. The VAS scores showed statistically significant relationships with the surgical method used (primary closure, nasolabial flap, or full-thickness skin graft).

The bilobed flap was the most frequently selected surgical method for nasal tip reconstructive procedures, followed by primary closure. The average VAS score for primary closure and bilobed flaps was 7, while the VAS for skin grafts was 5 (Table 3). Post-testing of the relationship between defect size and surgery method showed a significant difference in the choice among primary closure, bilobed flaps, and full-thickness skin grafts according to defect size. The VAS scores showed statistically significant differences according to the surgical method used (primary closure, full-thickness skin graft, or bilobed flap).

\section{DISCUSSION}

Nasal reconstruction is challenging for plastic surgeons due to the complex anatomical structure of the nose, its makeup of cartilage, and the lack of surrounding skin. Various considerations must be kept in mind when reconstructing defects after skin cancer resection. First, surgeons should consider the extent

Table 2. Classification of patient characteristics and results according to the surgical method used for the nasal ala

\begin{tabular}{lcccccc}
\hline Variable & Bilobed flap $(n=3)$ & Primary closure $(n=9)$ & Skin graft $(n=4)$ & Local flap $(n=14)$ & Nasolabial flap $(n=42)$ & $p$-value ${ }^{\text {a) }}$ \\
\hline Defect $(\mathrm{cm})$ & $1.5(1.2,2.2)$ & $0.7(0.7,0.8)$ & $2.4(2.1,2.8)$ & $1.7(1.3,2.0)$ & $2.3(2.1,3.0)$ & $<0.001$ \\
VAS & $8(8,9)$ & $5(5,5)$ & $4(3,4)$ & $6(6,7)$ & $7(6,7)$ & $<0.001$ \\
\hline
\end{tabular}

Values are presented as median (interquartile range).

VAS, visual analog scale.

a) $p$-value by the Kruskal-Wallis test.

Table 3. Classification of patient characteristics and results according to the surgical method used for the nasal tip

\begin{tabular}{|c|c|c|c|c|c|}
\hline Variable & Bilobed flap $(n=13)$ & Primary closure $(n=9)$ & Skin graft $(n=8)$ & Other flaps $(n=9)$ & $p$-value ${ }^{a)}$ \\
\hline Defect (cm) & $2(1.3,2.3)$ & $0.8(0.7,0.8)$ & $2.25(1.5,2.4)$ & $1.8(1.5,3.4)$ & 0.001 \\
\hline VAS & $7(7,7)$ & $7(7,8)$ & $5(4,5)$ & $6(5,6)$ & $<0.001$ \\
\hline
\end{tabular}

Values are presented as median (interquartile range).

VAS, visual analog scale.

${ }^{a} p$-value by the Kruskal-Wallis test. 
of skin cancer, whether it affects nearby tissues, the degree of loss of vital organs, the size and depth of the defect, and the possibility of complete resection.

Thin skin and cartilage are needed to reconstruct the shape of the nasal ala. Various surgical procedures, such as local flaps, distal flaps, skin grafts, and cartilage grafts, are available for this purpose. Although a previous study reported that nasal reconstruction with an auricular composite chondrocutaneous graft could yield the best aesthetic results [6], obstacles to using this method include differences in skin color, graft survival problems, and problems with the shape of the donor's ear. Since it was introduced by Blasius in 1848 as a method to reconstruct the ala, the V-Y flap has been widely used for nasal reconstruction. A V-Y advancement flap from the nasolabial area can be used to reconstruct an alar defect by moving a subcutaneous random flap into the defect area [7]. Color differences can be avoided by using the surrounding skin. Additionally, the V-Y advancement flap involves fewer problems at the donor site than other flaps. Thus, this method yielded excellent aesthetic results in this study. Among the methods used for alar reconstruction in this study, nasolabial local flaps-including V-Y advancement flaps and transposition flaps-yielded excellent aesthetic results (Figs. 1, 2). Although bilobed flaps showed similarly good results, they were used in only a small number of operations. Additionally, their VAS score was not significantly higher than those of other surgical methods. Local flaps of the random type using lateral skin and local flaps using the nasolabial fold showed similar results from an aesthetic point of view. In patients who underwent reconstruction with a local flap using the nasolabial fold area, the average size of the defect remaining after resection was larger than its size before the operation. Thus, nasolabial flaps were expected to have better results for alar reconstruction than random-type local flaps if the defect was large. Nasolabial flaps showed significantly better aesthetic results than primary closure or skin grafts, potentially because they may have avoided tip distortion, which occurred
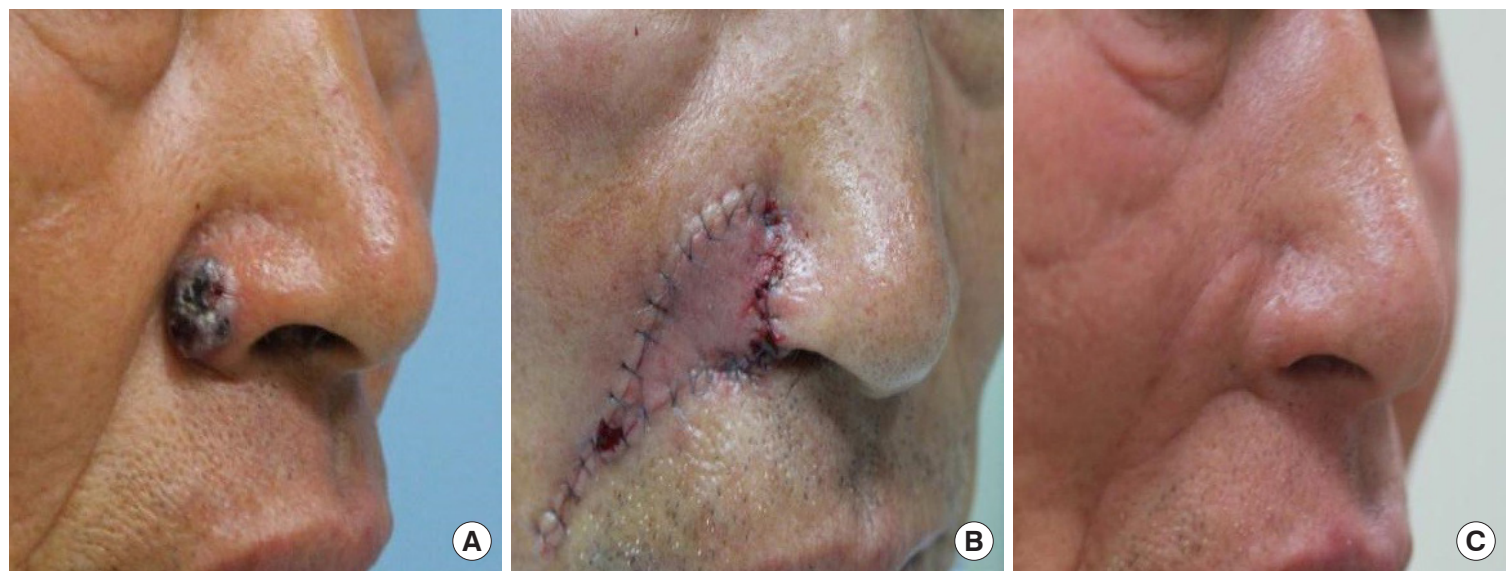

Fig. 1. A 70-year-old man was diagnosed with basal cell carcinoma in the nasal ala, and the defect was reconstructed with a nasolabial V-Y advancement flap after a wide excision. (A) Preoperative photograph. (B) Immediate postoperative photograph. (C) Photograph at 6 months after the operation. His visual analog scale score was 8.
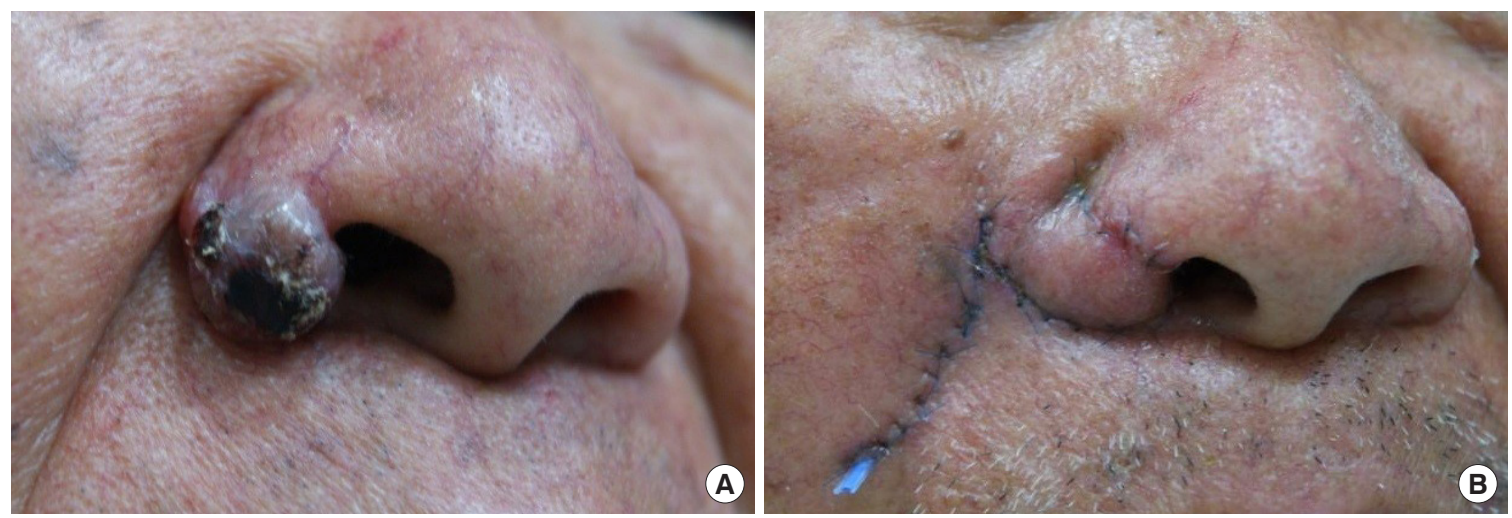

Fig. 2. An 84-year-old man was diagnosed with basal cell carcinoma in the nasal ala, and the defect was reconstructed with a nasolabial transposition flap after a wide excision. (A) Preoperative photograph. (B) Immediate postoperative photograph. His visual analog scale score was 7. 

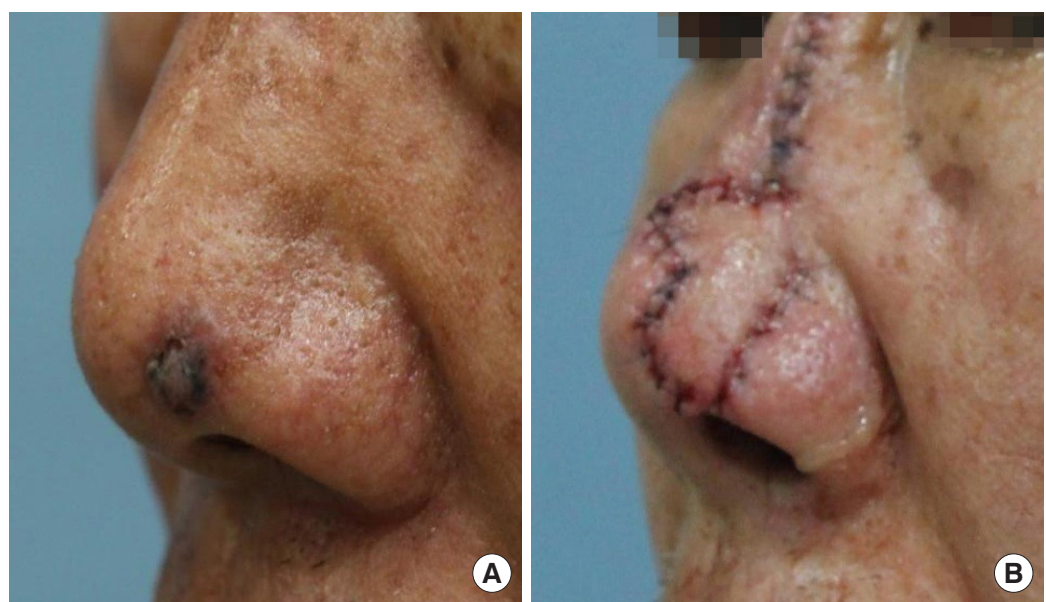

Fig. 3. A 75-year-old man was diagnosed with basal cell carcinoma in the nasal tip, and the defect was reconstructed with a bilobed flap after a wide excision. (A) Preoperative photograph. (B) Immediate postoperative photograph. His visual analog scale score was 7.

in primary closure procedures, and the problem of color mismatching observed with skin grafts. A bilobed flap may be useful for defects up to approximately $1.6 \mathrm{~cm}$. If the defect is larger than $2 \mathrm{~cm}$, a nasolabial flap may provide better aesthetic results.

Niranjan et al. [8] reported a successful case in which a defect of $2.5 \mathrm{~cm}$ near the nasal tip was reconstructed with a V-Y advancement flap from the nasal dorsum. Apostolou and Niranjan [9] reported another successful case in which a rotational $\mathrm{V}-\mathrm{Y}$ perforator flap was used to reconstruct a defect measuring $1.6 \mathrm{~cm}$ or more, yielding an excellent result. In this study, primary closure and bilobed flaps showed significantly better VAS scores than other surgical methods for the nasal tip. The defects treated with primary closure were mostly smaller than $1 \mathrm{~cm}$. If a defect exceeds $1.5 \mathrm{~cm}$ in size, a flap operation should be considered rather than primary closure. Bilobed flaps resulted in significantly higher VAS scores than other methods (Fig. 3). The reason for this is the insufficient amount of skin around the nasal tip, which made it difficult to use the surrounding skin as the donor. Local flaps, such as rotation flaps and V-Y advancement flaps using the surrounding skin, showed poor aesthetic results when the defect exceeded $1.5 \mathrm{~cm}$. If a defect exceeds $2 \mathrm{~cm}$, a skin graft may be considered. However, skin grafts showed the lowest VAS scores, indicating that they had poor aesthetic results. Madorsky and Ta [10] reported successful reconstruction in patients with defects at the nasal tip measuring less than $1.8 \mathrm{~cm}$. When the flap length was $1.5-1.8 \mathrm{~cm}$, distortion of the nasal tip occurred. However, the patients who underwent surgery with a bilobed flap in this study showed no distortion of the nasal tip.

Many restrictions must be kept in mind when selecting a surgical method. Cervelli et al. [11] reported some successful cases in which the nasal tip was reconstructed using median frontal, nasomental, or nasolabial flaps. However, approximately $21 \%$ of patients had unsatisfactory results due to tip retraction, which required reoperation in some cases. Consequently, primary closure is recommended if the defect is smaller than 1 $\mathrm{cm}$. If the defect size is $1-2 \mathrm{~cm}$, a bilobed flap should be considered. If the defect is $2 \mathrm{~cm}$ or larger, a nasolabial flap, forehead flap, or free flap is recommended.

This study has certain limitations. First, the number of patients was small. Second, long-term results could not be compared because long-term follow-up was not conducted. Additionally, there is an inherent limitation in evaluating surgical results using only two-dimensional postoperative photographs. More research is needed to evaluate the surgical outcomes over time and to assess which surgical methods offer better longterm results.

In conclusion, the results of this study demonstrate that nasolabial flaps showed excellent cosmetic results for reconstructing defects of the nasal ala, while primary closure and bilobed flaps yielded excellent results for reconstructing defects of the nasal tip.

\section{NOTES}

\section{Conflict of interest}

No potential conflict of interest relevant to this article was reported.

\section{Ethical approval}

The study was approved by the Institutional Review Board of Gyeongsang National University Hospital (IRB No. 2019-080002) and performed in accordance with the principles of the Declaration of Helsinki. Written informed consents were obtained. 


\section{Patient consent}

The patients provided written informed consent for the publication and the use of their images.

\section{ORCID}

Young Ji Park https://orcid.org/0000-0001-8416-7833

Gyu Hyeon Kwon https://orcid.org/0000-0002-8377-4698

Jun Oh Kim https://orcid.org/0000-0002-3860-6433

Woo Sang Ryu https://orcid.org/0000-0002-7472-3813

Kyung Suk Lee https://orcid.org/0000-0001-7731-1761

\section{REFERENCES}

1. Ujam AB, Bulstrode NW. A modified reconstructive technique for paediatric congenital alar rim deformity. Int J Pediatr Otorhinolaryngol 2019;118:201-5.

2. Lindsay KJ, Morton JD. Flap or graft: the best of both in nasal ala reconstruction. J Plast Reconstr Aesthet Surg 2015;68:13527.

3. Thomas DJ, King AR, Peat BG. Excision margins for nonmelanotic skin cancer. Plast Reconstr Surg 2003;112:57-63.

4. Nahhas AF, Scarbrough CA, Trotter S. A review of the global guidelines on surgical margins for nonmelanoma skin cancers. J Clin Aesthet Dermatol 2017;10:37-46.

5. Quinn JV, Drzewiecki AE, Stiell IG, Elmslie TJ. Appearance scales to measure cosmetic outcomes of healed lacerations. Am J Emerg Med 1995;13:229-31.

6. Jackson SM, Reisler T. Nasal reconstruction: nasal alar rim notching deformity reconstruction with auricular composite chondrocutaneous graft. Eplasty 2018;18:ic25.

7. Feinendegen DL, Langer MF, Roldan JC. Refinement of nasal reconstruction with a V/Y-alar-perforator flap. Plast Reconstr Surg Glob Open 2017;5:e1195.

8. Niranjan NS, Price RD, Govilkar P. Fascial feeder and perforator-based V-Y advancement flaps in the reconstruction of lower limb defects. Br J Plast Surg 2000;53:679-89.

9. Apostolou K, Niranjan NS. Lateral nasal artery perforator V-Y flap for reconstruction of nasal defects. J Plast Reconstr Aesthet Surg 2017;70:138-9.

10. Madorsky SJ, Ta A. Use of a lateral extended nasal island flap for nasal reconstruction. JAMA Facial Plast Surg 2019;21:4349.

11. Cervelli V, Bottini DJ, Gentile P. Reconstruction of the nasal tip. J Craniofac Surg 2007;18:1380-4. 